Association for Information Systems AIS Electronic Library (AISeL)

Wirtschaftsinformatik Proceedings 1999

Wirtschaftsinformatik

February 1999

\title{
Gestaltung von Verbesserungsprozessen: Workflow im Umfeld von Schulung und Ideenmanagement
}

Roland Rolles

Universität des Saarlandes, rolles@iwi.uni-sb.de

Yven Schmidt

Universität des Saarlandes, schmidt@iwi.uni-sb.de

August-Wilhelm Scheer

Universität des Saarlandes, scheer@iwi.uni-sb.de

Follow this and additional works at: http://aisel.aisnet.org/wi1999

\section{Recommended Citation}

Rolles, Roland; Schmidt, Yven; and Scheer, August-Wilhelm, "Gestaltung von Verbesserungsprozessen: Workflow im Umfeld von Schulung und Ideenmanagement" (1999). Wirtschaftsinformatik Proceedings 1999. 38.

http://aisel.aisnet.org/wi1999/38

This material is brought to you by the Wirtschaftsinformatik at AIS Electronic Library (AISeL). It has been accepted for inclusion in Wirtschaftsinformatik Proceedings 1999 by an authorized administrator of AIS Electronic Library (AISeL). For more information, please contact elibrary@aisnet.org. 


\section{Gestaltung von Verbesserungsprozessen: Workflow im Umfeld von Schulung und Ideenmanagement}

\section{Roland Rolles}

Universität des Saarlandes (rolles@iwi.uni-sb.de)

Yven Schmidt

Universität des Saarlandes (schmidt@iwi.uni-sb.de)

August-Wilhelm Scheer

Universität des Saarlandes (scheer@iwi.uni-sb.de)

\section{Inhalt}

1 Workflow Management, organisatorisches Lernen und kontinuierlicher Verbesserungsprozeß

2 Basisfaktoren der prototypischen Umsetzung

2.1 Intranet

2.2 Visualisierungstechniken

2.3 Schulungskonzepte

3 Lernen, Arbeiten, Vorschlagen, Verbessern - der Weg zu besseren Prozessen

3.1 Organisatorisches Lernen und kontinuierlicher Verbesserungsprozeß

3.2 Workflow-Nutzung und organisatorisches Lernen

3.3 Kontinuierlicher Verbesserungsprozeß und Workflow-Nutzung

4 Schulungssysteme und Verbesserungsobjekte - Eine Einordnung

4.1 Abstraktionsebenen der Schulung

4.2 Betrachtungsobjekte workflow-bezogener Verbesserungsaktivitäten

5 Fazit 


\begin{abstract}
Kontinuierliche Prozeßverbesserung, organisatorisches Lernen und Workflow Management sind aktuell diskutierte, innovative Konzepte. Diese in einem integrierten Ansatz miteinander in Einklang zu bringen, ist das Ziel des Prototypen KIWI (Kontinuierliche Prozeßverbesserung durch Integration von Workflow und Intranet). Als hypermediale Lernumgebung im Intranet richtet sich dieser an Mitarbeiter von Unternehmungen, die sich mit der Einführung und Nutzung eines Workflow-Management-Systems (WMS) befassen. Über KIWI werden dem Anwender neben dem Verständnis für eine prozeßorientierte Sichtweise und dem Umgang mit WMS auch Methoden und Werkzeuge eines kontinuierlichen Verbesserungsprozesses (KVP) vermittelt. Zum Zwecke einer ständigen Verbesserung von den der WorkflowAnwendung zugrundeliegenden Prozessen erlaubt KIWI zusätzlich die Durchführung dezentraler, modellbasierter Verbesserungsaktivitäten. Dadurch kann in der Unternehmung ein Kreislauf von Lernen, Arbeiten, Vorschlagen und Verbessern etabliert werden.

In diesem Beitrag werden Konzeption, Aufbau und Inhalte des Systems dargestellt. Darüber hinaus wird ein Ansatz zur Einordnung von Schulungssystemen vorgestellt. Dieser motiviert unterschiedliche Sichtweisen auf Schulungssysteme durch eine Betrachtung von Abstraktionsebenen. Anhand eines allgemeinen Geschäftsprozeßmodells wird zudem dargestellt, welche Arten von Verbesserungsobjekten bei workflow-bezogenen Prozeßverbesserungen untersucht werden müssen.
\end{abstract}

\title{
1 Workflow Management, organisatorisches Lernen und kontinuierlicher Verbesserungsprozeß
}

Die rasche Weiterentwicklung der Informationstechnik bringt für Unternehmungen vielfältige neue Anwendungsfelder mit sich. Vor diesem Hintergrund werden in Forschung und Praxis neue Konzepte erarbeitet, eingesetzt und evaluiert. Das vom BMBF geförderte Forschungsprojekt „Verbesserung von Geschäftsprozessen mit flexiblen Workflow-Management-Systemen (MOVE)“ [1] befaßt sich mit der Einführung und Nutzung von Workflow-Management-Systemen (WMS) (Herrmann et al. 1998a und 1998b). Neben Konzepten zur flexiblen Einsetzbarkeit von WMS und Vorgehensweisen zur Beteiligung der Mitarbeiter in den einzelnen Projektphasen steht dabei die Etablierung eines kontinuierlichen Verbesserungsprozesses (KVP) in den beteiligten Betrieben im Vordergrund. Ein entscheidender Faktor für einen erfolgreichen Workflow-Einsatz und den KVP ist das organisatorische Lernen: Mitarbeiter müssen einerseits geschult werden, um das notwendige Prozeßverständnis zu erlangen, mit der neuartigen Vorgangsbearbeitungssoftware umgehen zu können und Wissen über Ziele und Methoden eines Verbesserungsprozesses zu erwerben, und sollen andererseits wieder Wissen an die Organisation 
in Form von Verbesserungsideen und -vorschlägen zurückgeben. Durch diesen Wissensrückfluß wird die organisatorische Wissensbasis ständig angereichert und aktualisiert. Hierdurch wird das organisatorische Lernen (im Verständnis von Oberschulte 1994) ermöglicht. Am Institut für Wirtschaftsinformatik (IWi) der Universität des Saarlandes wurde im Rahmen von MOVE daher der Prototyp KIWI (Kontinuierliche Prozeßverbesserung durch Integration von Workflow und Intranet) entwickelt. Dieser erlaubt sowohl eine erste Qualifizierung der Mitarbeiter für den Umgang mit einer Workflow-Anwendung als auch eine ständige Weiterbildung im Sinne eines Training-on-the-job und ist somit als Baustein des Total Quality Learning (Schnauber et al. 1997) einsetzbar.

In Abschnitt 2 werden zunächst die Bausteine bzw. Basisfaktoren von KIWI dargestellt. Als Plattform wurde das Intranet gewählt, da es eine leichte Zugänglichkeit mit intuitiven Navigations- und Visualisierungstechniken verknüpft (vgl. Abschnitt 2.1). Auf die in KIWI zugrundegelegten Visualisierungstechniken geht Abschnitt 2.2 ein, eine Beschreibung der verwendeten Schulungskonzepte erfolgt in Abschnitt 2.3.

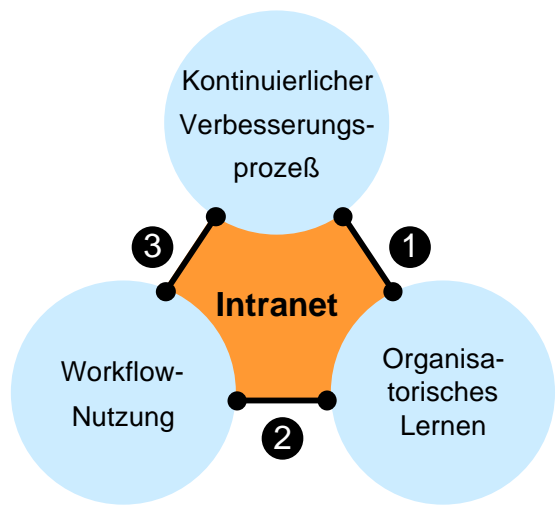

Abbildung 1: Bausteine und Schnittstellen des Gesamtkonzepts

Abbildung 1 visualisiert den Möglichkeitenraum von KIWI, der sich über Workflow-Nutzung, kontinuierlichem Verbesserungsprozeß und organisatorischem Lernen aufspannt. Es ergeben sich somit 3 Anwendungsfelder, für die KIWI Lösungen anbietet: Das Erlernen der Methoden und Werkzeuge eines kontinuierlichen Verbesserungsprozesses (Schnittstelle 1: wird in Abschnitt 3.1 behandelt), intensive Vorbereitung auf die Workflow-Nutzung (Schnittstelle 2: Abschnitt 3.2) sowie das Anwenden der erlernten Methoden im workflow-gestützten, kontinuierlichen Verbesserungsprozeß (Schnittstelle 3: Abschnitt 3.3).

In Abschnitt 4 werden Schulungssysteme und Verbesserungsobjekte wissenschaftlich systematisiert und die entwickelte Architektur als Grundlage für die Einordnung von KIWI herangezogen. Das hierbei entwickelte Rahmenwerk eignet sich dabei nicht nur zur Einordnung von Schulungssystemen, sondern kann gleichermaßen als Architektur zu deren Entwicklung herangezogen werden. 


\section{Basisfaktoren der prototypischen Umsetzung}

Zentraler Baustein des Ansatzes ist eine computerbasierte, integrierte Lernumgebung, die sowohl den Grundstein für die Nutzung der Workflow-Anwendung legt, als auch für die Beteiligung am kontinuierlichen Verbesserungsprozeß die Voraussetzungen schafft. Diese muß so gestaltet sein, daß die Motivation zur Nutzung des Systems hoch ist. Mit KIWI wurde eine Lernumgebung geschaffen, in der sich der Benutzer ungezwungen bewegen kann und spielerisch ohne das Gefühl von Zwang und Kontrolle den Umgang mit seiner veränderten Arbeitsumgebung und die Partizipation am KVP erlernt.

\subsection{Intranet}

Kontinuierliche Lern- und Verbesserungsprozesse lassen sich im UnternehmungsIntranet in idealer Weise miteinander verknüpfen. Dies liegt an der besonderen Eignung der Intranet-Technologie, mit deren Einsatz Nutzenpotentiale sowohl quantitativer als auch qualitativer Art verbunden sind (Döge 1997, S. 44-45). Hierunter fallen auf qualitativer Seite u.a. die erhöhte Mitarbeitermotivation durch einen erleichterten Informationszugriff und eine verbesserte Informationsverfügbarkeit, eine Vereinheitlichung von Abläufen sowie eine Reduktion telefonischer Rückfragen zwecks Informationsbeschaffung. Quantitativ meßbarer Nutzen entsteht beispielsweise durch die Plattformunabhängigkeit der Infrastruktur, durch Einsparungen aufgrund reduzierter Papierverwaltung sowie durch Effizienzsteigerung wegen verbesserter Lerneffekte. Auf diese Weise kann ein KVP ideal in den täglichen Arbeitsprozeß integriert werden und muß nicht weiter losgelöst von diesem organisiert werden, wie es bei traditionellen Verfahren wie dem betrieblichen Vorschlagswesen oder Qualitätszirkeln momentan noch der Fall ist.

\subsection{Visualisierungstechniken}

Da für den Mensch die Aufnahme (audio-)visueller Informationen im Vergleich zu rein textuellen Präsentationsformen erheblich leichter ist (Jain 1997), wurden für die Vermittlung der Inhalte vielfältige Visualisierungstechniken verwendet:

- Hypertext: Über Hypertextlinks wurden logisch zusammengehörende Schulungsteile durch Verweise miteinander verknüpft. So können didaktisch gut aufgebaute Lerneinheiten angeboten werden.

- Bilder: Zur besseren Visualisierung von Inhalten wird der Text durch Bilder ergänzt, die zur Illustration von Sachverhalten geeignet sind.

- Animationen: Bewegte Bilder bringen dem Benutzer nicht-statische Sachverhalte näher, wie etwa das Wandern von Arbeitsmappen über Bearbeiter.

- Modelle: Geschäftsprozeß- bzw. Workflow-Modelle spielen in KIWI eine zentrale Rolle. Beim Lernen des Basiswissens zu WMS sind sie Betrachtungsgegenstand und im Verlauf des KVPs werden sie als Objekt von Verbesserungsvorschlägen herangezogen.

- Screen-Shots: Durch die Wiedergabe einer Momentaufnahme einer Benutzungsoberfläche wird im Gegensatz zu einer subjektiven verbalen Beschrei- 
bung von Bildschirminhalten eine photogenaue, objektive Wiedergabe ebendieser ermöglicht.

- Screen-Cams: Screen-Cams sind audio-visuell und ermöglichen die visuelle Wiedergabe von Bildschirmaktivitäten, die in einer Audiospur auch auskommentiert werden können. Die Einzelbilder (frames) einer Screen-CamPräsentation stellen wiederum Screen-Shots dar.

- Showcases (Walter 1998): Videoclips, die einen Ausschnitt aus einem interessierenden Umfeld visualisieren und in einen bestimmten Gesamtzusammenhang einordnen. Diese können einem Benutzer beispielsweise Ausschnitte aus aktuellen oder zukünftigen Tätigkeiten präsentieren.

Der Einsatz dieser Visualisierungstechniken zum erfolgreichen Lernen wird auch in den Abschnitten 3.1 und 3.2 noch einmal näher beleuchtet.

\subsection{Schulungskonzepte}

In KIWI wurden darüber hinaus mehrere verschiedene Schulungskonzepte integriert, um für den Benutzer eine möglichst vielfältige Lernumgebung zu schaffen. Hier wurde je nach zu vermittelndem Inhalt und Zielsetzung eine geeignete Form gewählt, wie man den Benutzer mit dem Lernstoff konfrontiert. Bei der Verfolgung eines Schulungskonzeptes können auch mehrere der in Abschnitt 2.2 genannten Visualisierungstechniken angewendet werden. Im einzelnen wurden über die Einstellung „,normaler“ HTML-Seiten mit Schulungsinhalten folgende darüber hinausgehende Schulungskonzepte in KIWI verwirklicht:

- Geführte Tour: Die geführte Tour ermöglicht einem neuen Benutzer, sich einen Überblick über das System zu verschaffen. Hierzu navigiert er auf einem vorbestimmten Weg über verschiedene Inhalte von KIWI und erhält so einen Eindruck über das Gesamtsystem. Zusätzlich zur Vermittlung der „essentials“ zu Prozeßorientierung, Nutzung von WMS und zu KVP erhält der Benutzer auch Hinweise auf weitere Features von KIWI. Zielsetzung der Anwendung dieses Schulungskonzeptes ist nicht der Aufbau detaillierten, tiefgründigen Wissens beim Anwender, sondern die Schaffung eines Überblicks auch bei knappen Zeitressourcen. (Inhalt: Überblick über alle Teilbereiche von KIWI, Ziel: schlaglichtartige Übersicht bei geringem Zeitbedarf).

- Interaktive Fallstudien: Mittels einer interaktiven Fallstudie wird dem Benutzer die Anwendung von gelerntem Wissen verdeutlicht und Interaktion des Benutzers dahingehend erfordert, daß diesem vom System Aufgaben gestellt werden und das System je nach dessen Aufgabenlösung auf unterschiedliche Weise reagiert. Im einfachsten Fall wird hier vom Benutzer beispielsweise die Auswahl der richtigen Lösungsalternative bei einer Multiple-Choice-Frage gefordert. In KIWI wurde eine interaktive Fallstudie benutzt, um die Anwendung des KVP anhand eines durchgängigen Beispiels zu erlernen. (Inhalt: Anwendung des KVP, Ziel: Learning by doing).

- Demonstration: Eine Demonstration gibt in bewegten Bildern oder per persönlicher Präsentation den Ablauf eines Sachverhaltes, z.B. die Lösung eines Problems wieder. In KIWI wurde ein durchgängiges Beispiel einer KVPSitzung (vgl. auch Abschnitt 3.1) mit entsprechenden Videosequenzen aufbe- 
reitet eingestellt, um den Benutzer den beispielhaften Ablauf einer solchen Sitzung näherzubringen (Inhalt: Anwendung des KVP, Ziel: Veranschaulichung).

- Glossar: Das Glossar ermöglicht die Anzeige von erklärenden Texten zu ausgewählten Fachbegriffen. Per Fly-over-help ist somit die Anzeige eines Hilfetextes zu ausgewählten komplizierten Begriffen möglich. Bei Verweilen des Mauscursors auf dem Glossarbegriff wird die hinterlegte Erklärung aktiviert. (Inhalt: Erklärung ausgewählter Fachbegriffe, Ziel: Erhöhung des Verständnisses).

- Suche: Die Suche ermöglicht das Auffinden von themenverwandten Informationen zu einem bestimmten, vom Benutzer einzugebenden Suchbegriff per Suche über alle KIWI-Inhalte. (Inhalt: alles, Ziel: Selektion und Vertiefung ausgewählter Inhalte).

- FAQs: In den FAQs werden ausgewählte, immer wieder auftauchende Fragen gesammelt und beantwortet (Inhalt: immer wieder gestellte Fragen zu allen Themenbereichen, Ziel: effiziente Problemlösung durch Rückgriff auf bereits vorliegendes Wissen).

\section{Lernen, Arbeiten, Vorschlagen, Verbessern - der Weg zu besseren Prozessen}

Für die kontinuierliche Verbesserung workflow-gestützter Geschäftsprozesse wurde bereits in frühen Phasen des MOVE-Projekts eine Vorgehensweise erarbeitet (Rolles 1998). Der Prototyp KIWI stellt eine konsequente Weiterentwicklung und Umsetzung dieses Vorschlags dar. Er geht von der Grundannahme eines Strebens nach stetigen Verbesserungen im Betrieb aus. Dieses Streben manifestiert sich in einer bestimmten Abfolge unterschiedlicher Tätigkeiten (vgl. Abbildung 2).

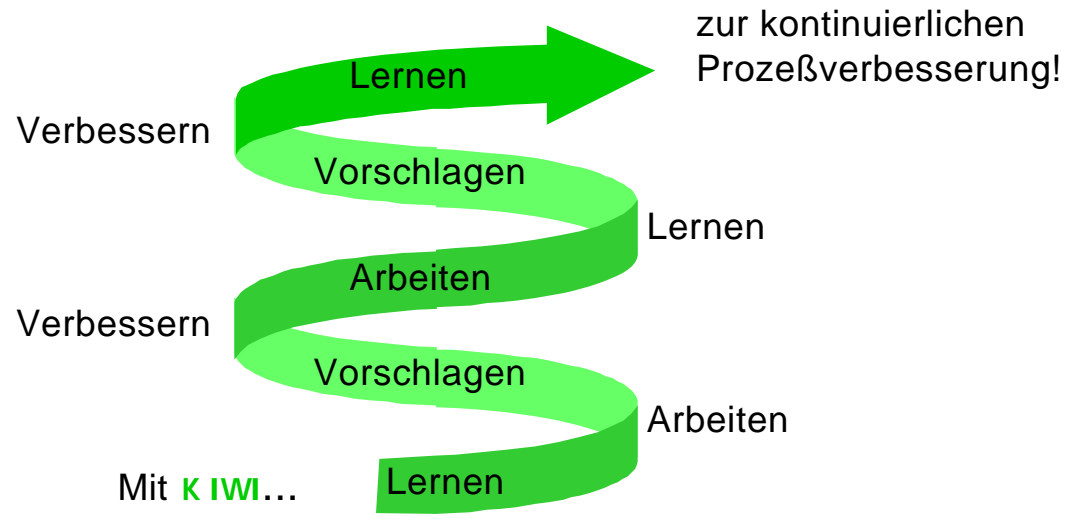

Abbildung 2: Der Weg zur kontinuierlichen Prozeßverbesserung 
Am Anfang steht dabei das Lernen, das dem einzelnen Mitarbeiter das Bewegen in seiner durch Workflow Management neuen Arbeitswelt erleichtert. Auch das Erlernen von Kreativitätstechniken und methodischen Vorgehensweisen zur Problemlösung sind dieser Phase zuzuordnen. Das Lernen sollte nie aufhören, sondern ständiger Wegbegleiter bei den Arbeitsprozessen sein. Beim Arbeiten mit der Workflow-Anwendung wird das Erlernte umgesetzt. In dieser Phase stellt der Mitarbeiter Probleme in der Organisation, Schwachstellen in der Anwendung etc. fest. Diese dienen als Grundlage zur Formulierung von Verbesserungsideen, dem Vorschlagen. Die Umsetzung der Vorschläge führt schließlich zu einer Verbesserung von Prozessen und Anwendung und der beschriebene Zyklus beginnt von vorne.

\subsection{Organisatorisches Lernen und kontinuierlicher Verbesserungsprozeß}

Durch Verbesserungsvorschläge können Mitarbeiter am Arbeitsplatz an der Verbesserung ihrer betrieblichen Situation mitwirken. Mit jeder Umsetzung eines Verbesserungsvorschlages wird quasi ein neuer Standard definiert (Imai 1994, S. 37f.), der nach Umsetzung eines neuen Verbesserungsvorschlages durch einen wieder neuen Standard ersetzt wird. Da sich dieser Zyklus immer wiederholt, spricht man auch von einem kontinuierlichen Verbesserungsprozeß. Die zugrundeliegende Ver-

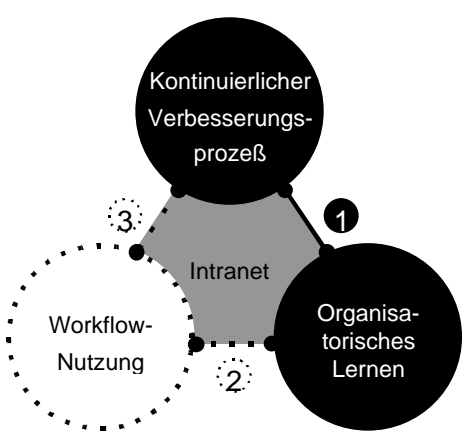
besserungsmentalität stützt sich also in erster Linie auf das Wissen und die Kreatvität der Mitarbeiter (Jacobi 1997, S. 9).

Als Basisbausteine, von denen das erfolgreiche Gelingen kontinuierlicher Verbesserungsprozesse abhängt, können Motivation, Schulung, Wissen und Kreativität aufgefaßt werden. In der Literatur werden im Zusammenhang mit der Wirksamkeit des Verbesserungswesens sog. Barrieren diskutiert, deren Überwindung die Grundvoraussetzung für das Ausschöpfen von Optimierungspotentialen darstellt (Thom 1991, S. 43ff). Eine dieser Barrieren ist die sogenannte Fähigkeitsbarriere, die ausschlaggebende Ursache dafür sein kann, daß ein einzelner Mitarbeiter nicht am Verbesserungswesen teilnehmen kann (für eine detailliertere Untersuchung von Fähigkeits- und Willensbarrieren vgl. Hagemeyer et al. 1998b). Das Problem des Nicht-Könnens ist mit einer entsprechenden Qualifizierung der Mitarbeiter zu beheben. Sie müssen sich das Wissen aneignen, das bei der Durchführung kontinuierlicher Verbesserungsprozesse von Relevanz ist. Insbesondere methodisches Wissen, konzeptionelles Wissen sowie Prozeßwissen ist hierbei von Bedeutung (für eine Kategorisierung von Wissensarten vgl. von Krogh/Venzin 1995).

Die Vermittlung des relevanten Wissens für den kontinuierlichen Verbesserungsprozeß erfolgt in KIWI in der Lerneinheit KVP. Dort wird dem Mitarbeiter ein Grundverständnis für die Notwendigkeit zur Durchführung eines kontinuierlichen 
Verbesserungsprozesses sowie entsprechendes Methodenwissen vermittelt. Zunächst wird jedoch motiviert, welche Idee hinter dem Begriff KVP steht. Weitere Lerneinheiten setzen sich mit dem Ablauf eines KVP, der Schwachstellenanalyse von Prozessen, Sollprozeßentwicklung sowie Werkzeugen für den KVP auseinander.

Um nicht nur Wissen darzustellen und zu reproduzieren soll dem Mitarbeiter auch die Anwendung dieses Wissens demonstriert werden und ihm die Möglichkeit gegeben werden, das gelernte Wissen beispielhaft selbst in einer Fallstudie anzuwenden.

Abbildung 3 zeigt einen Ausschnitt der Demonstration des Ablaufes einer KVPSitzung in KIWI. Hier wird die Anwendung der vorher gelernten Methoden eingehend veranschaulicht. Diese beispielhafte KVP-Sitzung wurde didaktisch in Untereinheiten zerlegt. Zu jeder Phase dieser Sitzung werden textuell Hintergrundinformationen geliefert, ein möglicher Ablauf dieser Phase wird in Form einer kurzen Videoanimation visualisiert. So gibt es Showcases, die den Ablauf von Workshops zur Modellierung von Sollkonzepten visualisieren oder auch Videos, die den Umgang mit Werkzeugen zur Kreativität wie Ishikawa-Diagramm (Ishikawa 1985) an einem Beispiel transparent machen.

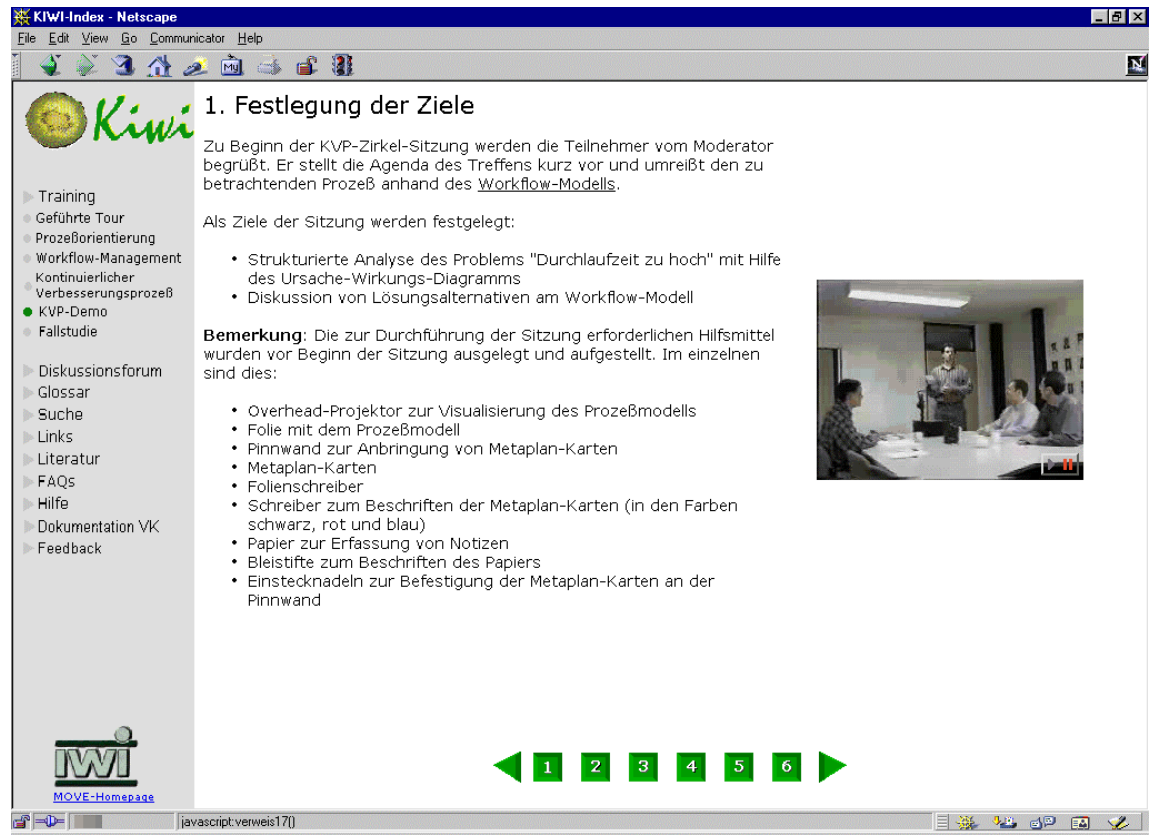

Abbildung 3: Demonstration einer KVP-Sitzung in KIWI

In einer Fallstudie wird der Benutzer durch den kompletten Verbesserungszyklus von der Analyse eines bestehenden Prozesses über die Sollkonzeption und Implementierung bis hin zur Anwendung des verbesserten Prozesses geführt. An geeig- 
neten Stellen hat er Entscheidungen zu treffen, die er auch so bei der tatsächlichen Nutzung der Workflow-Anwendung zu treffen hat. Er wird auf Fehler hingewiesen und bekommt Hinweise und Ratschläge. Somit kann er spielerisch das Verständnis für die neue Technologie und die mit ihr verbundenen Vorgehensweisen erlangen, so daß hiermit gleichzeitig eine Verbindung zu Lernen und Workflow gegeben ist.

\subsection{Workflow-Nutzung und organisatorisches Lernen}

Die unter 2.1 genannten Barrieren lassen sich natürlich auch auf diesen Ausschnitt der Betrachtung übertragen. So gilt es, Fähigkeitsbarrieren durch gezielte Schulung und Vermittlung des relevanten Basiswissens abzubauen sowie Ängste und Vorbehalte gegenüber der neuen Technologie durch eine behutsame, spielerische Vermittlung der Lerninhalte auszuräumen. In KIWI werden daher in der Lerneinheit Prozeßorientierung die Mitarbeiter zunächst behutsam mit dem Prozeßgedanken vertraut

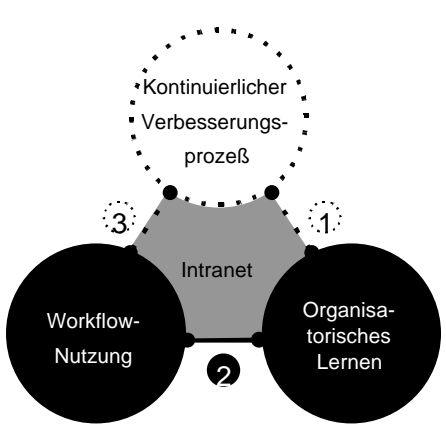
vertraut gemacht und der Übergang von einer funktionsorientierten zu einer prozeßorientierten Sichtweise motiviert. Dies ist für einen erfolgreichen WorkflowEinsatz unentbehrlich, da die Neuordnung von Kompetenzen, Verantwortung und Aufgaben von den Mitarbeitern getragen werden muß (Gaitanides et al. 1994, S. 2 ff.).

Erst in der Folgelerneinheit Workflow Management wird der Benutzer mit der Technologie des Workflow Managements und der Arbeit mit WMS vertraut gemacht. Hierzu werden zunächst Grundwissen über WMS und Voraussetzungen für den Einsatz von WMS vermittelt. Weitere Unterlerneinheiten fokussieren darauf, welche Prozesse für eine Unterstützung mit WMS geeignet sind, welche Vorteile der Einsatz von WMS bietet, wie die Einführung von WMS abläuft und wie sich die Arbeitsumgebung beim Einsatz von WMS ändert. Abbildung 4 gibt die Lerneinheit zum Thema Workflow Management sowie die Unterteilung in Unterlerneinheiten wieder. Zur Auflockerung wird auch eine Animation eingesetzt, um zu visualisieren, wie ein Dokument über verschiedene Bearbeiter wandert.

In der Unterlerneinheit „Wie arbeitet man mit einem WMS“ werden Screen-Shots verwendet, um die Arbeit mit der Workflow-Anwendung anhand von Aufnahmen der entsprechenden Bildschirmmasken zu visualisieren. Zusätzlich wird die Arbeit mit WMS anhand eines durchgängigen Beispiels von der Anmeldung am System bis hin zur Nutzung der Applikationen anhand von Screen-Cam-Präsentationen eingängig verdeutlicht. 


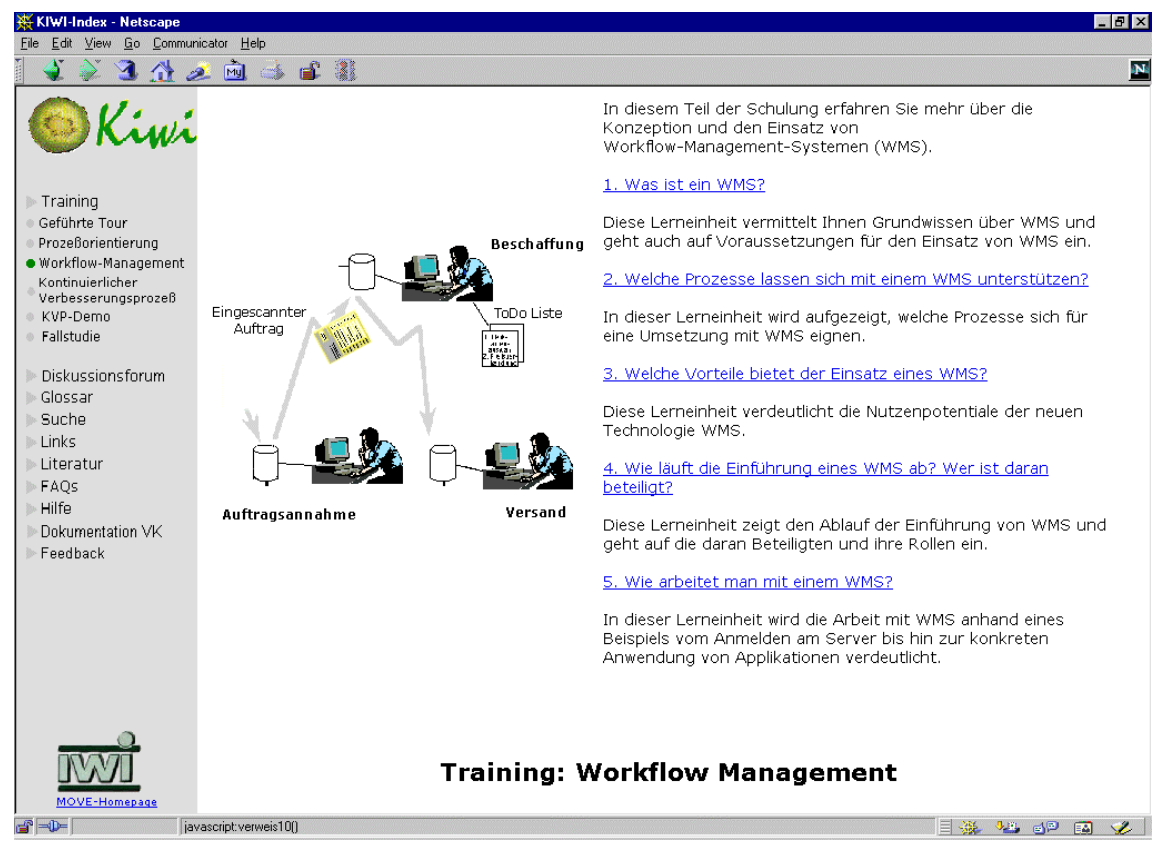

Abbildung 4: Lerneinheit Workflow Management

Auch auf die dem Beispiel zugrundeliegenden Geschäftsprozeßmodelle wird ausführlich anhand weiterer Screen-Cam-Präsentationen eingegangen. Bei der Nutzung von Geschäftsprozeß- und Workflow-Modellen ist dabei besonders behutsam vorzugehen, da i.d.R. bei einem Großteil der Anwender keine Methodenkenntnis bzgl. der verwendeten Beschreibungssprachen vorliegt (Hagemeyer/Rolles 1998). Daher ist es ratsam, die Modelle jeweils in Kombination mit weiteren, zusätzlich beschreibenden Visualisierungsformen zu verwenden, um die Verständlichkeit und Akzeptanz zu steigern.

\subsection{Kontinuierlicher Verbesserungsprozeß und Workflow- Nutzung}

Durch das kontinuierliche Lernen wird der Anwender in die Lage versetzt, sich kritisch mit Organisationsstruktur und WorkflowAnwendung auseinanderzusetzen. Er wird für Schwachstellen sensibilisiert und besitzt Wissen über den Einsatz von KVP-Werkzeugen und Kreativitätstechniken wie etwa Mind Mapping (Eipper 1998), die Problemlösungswerkzeuge von Kaizen (Imai 1994) oder Methoden der systematischen Strukturierung (Mehrmann

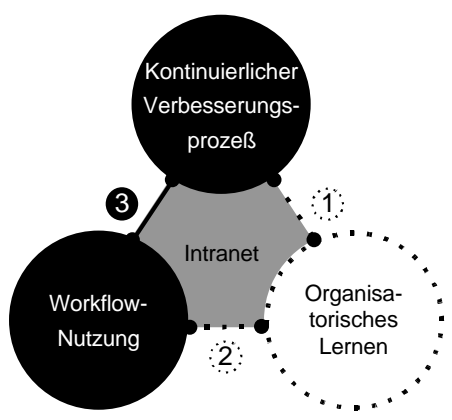


1994). Diese in Verbindung mit einem dezentralen Verbesserungsvorschlagswesen einsetzbar zu machen, ist Ziel von KIWI. Zu diesem Zweck werden unterschiedliche Hilfsmittel angeboten und ein solcher KVP methodisch unterstützt.

Die zugrundegelegte Vorgehensweise inklusive der eingesetzten Werkzeuge stellt Abbildung 5 dar. KIWI unterstützt die Anwender dabei, Ansprechpartner für ihre Verbesserungsvorschläge zu ermitteln, ihre Vorschläge per E-Mail einzureichen und mit Kollegen zu diskutieren. Durch die Integration mit dem eingesetzten WMS kann der Anwender im Intranet auf die Workflow-Modelle zugreifen. Verbesserungsvorschläge können an diesen vermerkt und bei Bedarf über gemeinsame Diskussion am Modell (via Shared Browsing, d.h. das gemeinsame Navigieren durch Webseiten, und Videoconferencing) verfeinert werden. Der Prozeßverantwortliche als Empfänger von Verbesserungsvorschlägen prüft diese und setzt sie gegebenenfalls unmittelbar im Workflow-Modell um. Auf einem Schwarzen Brett werden Statistiken zu eingegangenen und umgesetzten Verbesserungsvorschlägen veröffentlicht.

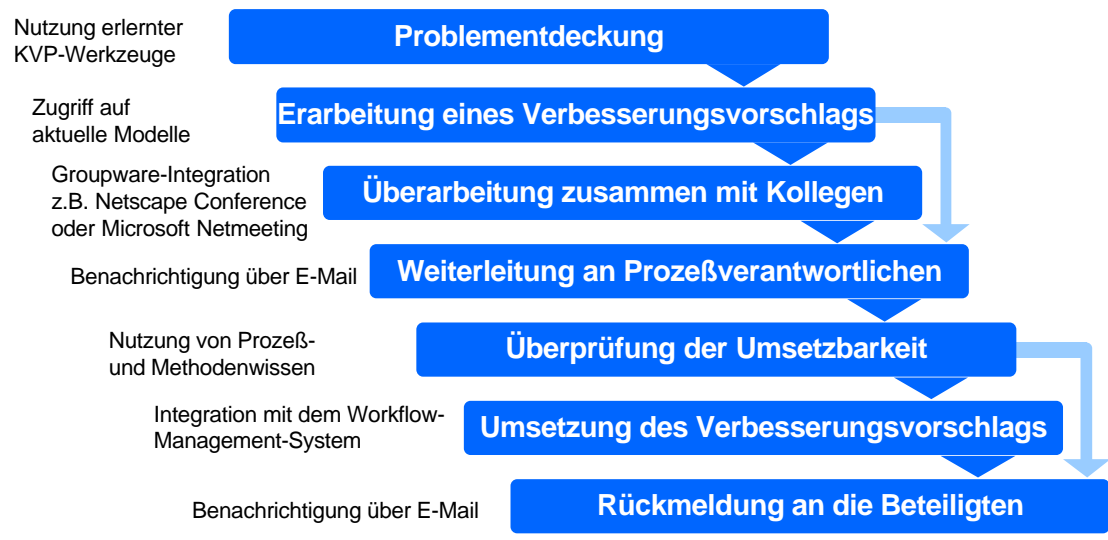

Abbildung 5: Vorgehensmodell zur dezentralen Prozeßverbesserung

Abbildung 6 visualisiert, auf welche Art und Weise die Nutzung dieses KVPBausteins erfolgt. Es handelt sich um eine modellbasierte Vorgehensweise, bei der über einen entsprechenden Navigationsbaum (linker Teil der Benutzungsoberfläche) das gewünschte Modell anhand des Geschäftsprozeßkontextes vom Vorschlagseinreicher ausgewählt werden kann. Im vorliegenden Fall ist das Prozeßmodell der Vorkalkulation Gegenstand eines Verbesserungsvorschlags. Bei Auswahl des Modells wird der zuständige Prozeßverantwortliche direkt mitgeliefert. Der weitere Ablauf nach Einreichung erfolgt wie oben kurz beschrieben. Neben der Visualisierung durch Modelle ist im weiteren Projektverlauf auch daran gedacht, z.B. Screen-Shots der Bildschirmmasken der operativen Anwendungen in das System einzustellen, um diese gleichermaßen zum Objekt für Verbesserungsaktivitäten zu machen. 


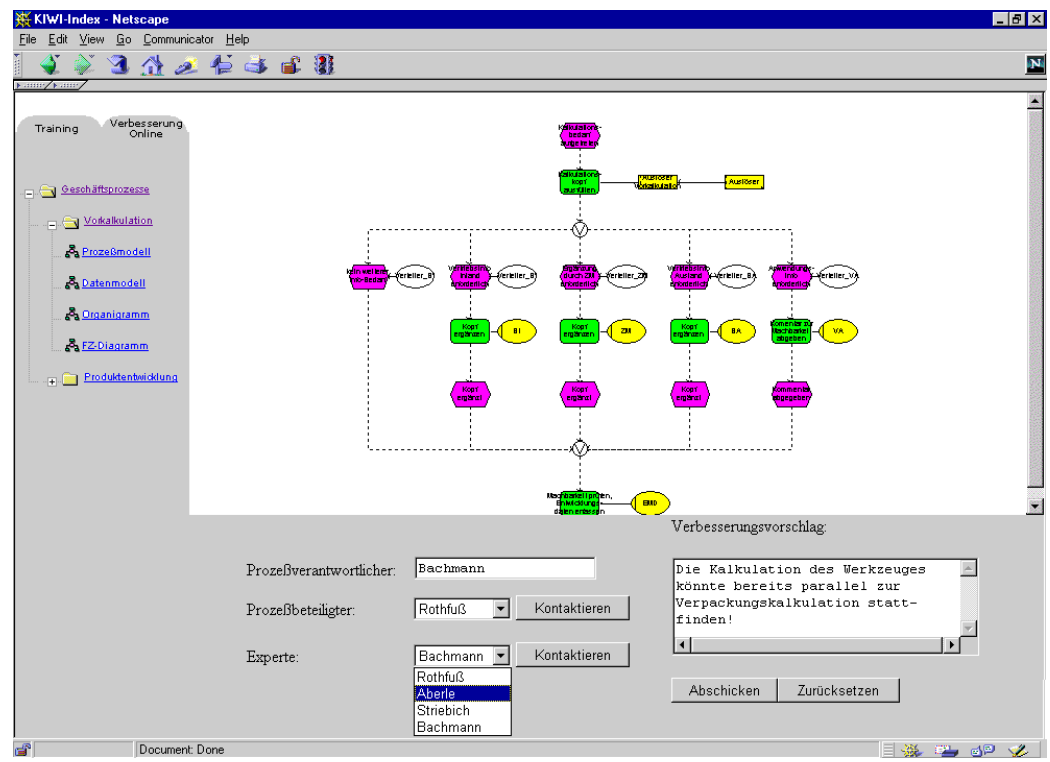

Abbildung 6: Modellbasiertes Verbesserungsvorschlagswesen

\section{Schulungssysteme und Verbesserungsobjekte - Eine Einordnung}

Nachdem die Zusammenhänge zwischen organisatorischem Lernen, kontinuierlichem Verbesserungsprozeß und Workflow-Nutzung in den vorangegangenen Abschnitten dargestellt wurden, erfolgt nun eine Einordnung von Schulungssystemen und Verbesserungsobjekten. Diese führt die bisherigen Ergebnisse zusammen und stellt zugleich einen Rahmen für die Betrachtung von Schulungsund Verbesserungsmaßnahmen dar. $\mathrm{Zu}$ diesem Zweck wird in Abschnitt 4.1 ausgearbeitet, inwiefern Schulungssysteme sich anhand von Abstraktionsebenen beschreiben lassen. Abschnitt 4.2 leitet anhand eines allgemeinen Geschäftsprozeßmodells her, welche Betrachtungsobjekte Gegenstand betrieblicher Verbesserungsaktivitäten im Rahmen der Workflow-Nutzung sind.

\subsection{Abstraktionsebenen der Schulung}

Scheer unterscheidet bei der Betrachtung von Geschäftsprozeßmodellen zwischen vier Abstraktionsebenen: Ausprägungs-, Anwendungs-, Meta- und Meta ${ }^{2}$-Ebene (Scheer 1998, S. 26-31). Demnach können Geschäftsprozesse je nach Nutzungsziel auf unterschiedlichen Abstraktionsniveaus modelliert werden. Möchte man beispielsweise einen individuellen Auftragsbearbeitungsprozeß beschreiben, so werden konkrete Ausprägungen wie z.B. der Name des Kunden oder die Nummer 
des bestellten Artikels modelliert. Wird von den Ausprägungen eines konkreten Falls abstrahiert, so beschreibt man einen Geschäftsprozeßtyp und befindet sich damit auf Anwendungsebene. Beim Übergang zur Meta-Ebene wird von Anwendungsinhalten abstrahiert. Das Resultat ist ein allgemeines Geschäftsprozeßmodell (als Abstraktion von Geschäftsprozeßtypen wie z.B. Auftragsabwicklung oder Produktentwicklung) (vgl. auch Abschnitt 4.2), welches die Menge der zur Beschreibung von Geschäftsprozessen nötigen Konstrukte festlegt. Ein derartiges Meta-Modell gibt also an, aus welchen Bausteinen sich Modelle zu Geschäftsprozeßtypen (Anwendungsebene) und damit auch Geschäftsprozeßinstanzen (Ausprägungsebene) zusammensetzen. Setzt man diesen Abstraktionsprozeß weiter fort, so gelangt man durch eine Abstraktion von Modellierungsinhalten zur Meta ${ }^{2}$ Ebene.

In Analogie hierzu lassen sich auch Schulungssysteme anhand der genannten Abstraktionsebenen beschreiben. Dies gibt insbesondere Aufschluß über eingesetzte Schulungskonzepte und zu vermittelnde Schulungsinhalte. Für die Betrachtungsobjekte des oben beschriebenen Schulungssystems KIWI zeigt Abbildung 7 die Abstraktionsebenen der Schulung. Die Abbildung stellt nicht alle vorhandenen Beziehungen komplett dar, sondern zeigt am Fall des Workflow Managements in der Vorkalkulation exemplarisch die Unterteilung in die vier Ebenen.

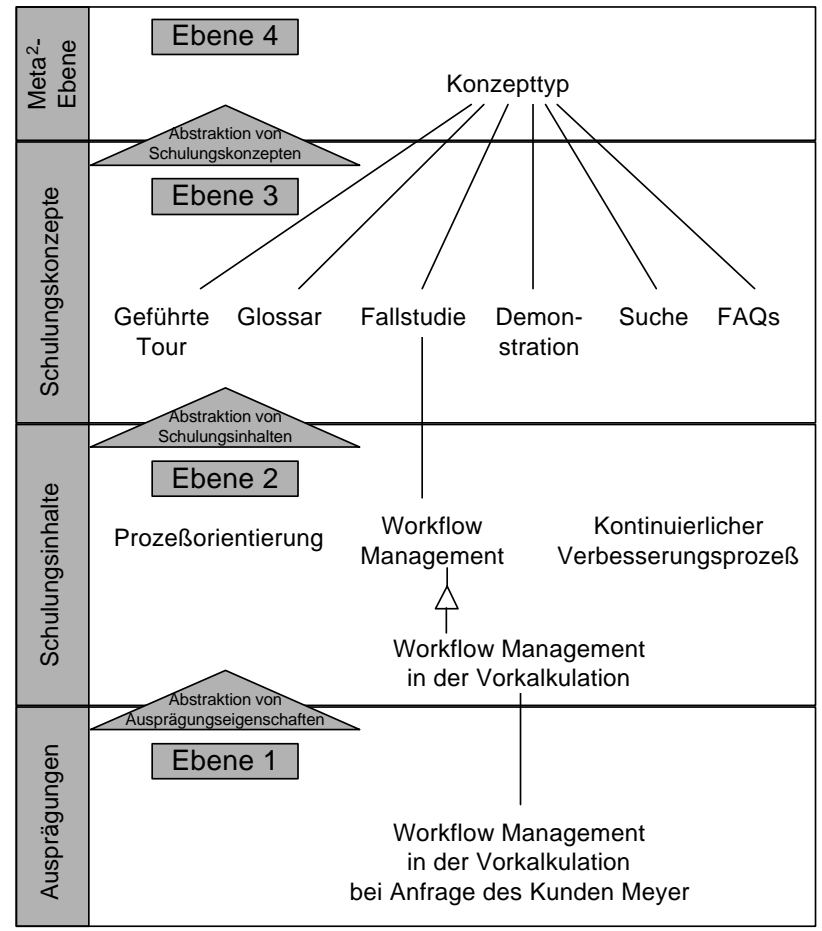

Abbildung 7: Abstraktionsebenen der Schulung am Beispiel von KIWI (in Anlehnung an Scheer 1998) 
Die Ebenen 1 bis 4 beschreiben damit die folgenden Sachverhalte.

- Ebene 1 (Ausprägungen): Schult man Mitarbeiter auf der Ebene von Ausprägungen, so sind konkrete Fälle Lerngegenstand. Ein Lernprogramm zum Umgang mit der Drehmaschine D17 oder zum Erlernen des Textverarbeitungsprogramms Word sind Beispiele für diese Ebene.

- Ebene 2 (Schulungsinhalte): Sollen nicht individuelle Gegebenheiten im Mittelpunkt der Schulung stehen, sondern allgemeine Anwendungsinhalte, so ist ein Schulungssystem mit Inhalten der Ebene 2 heranzuziehen. Geschult würden also - um bei den genannten Beispielen zu bleiben - der Umgang mit Drehmaschinen aller Art oder Textverarbeitungssystemen im allgemeinen.

- Ebene 3 (Schulungskonzepte): Hier steht die Frage im Mittelpunkt, in welcher Form die Inhalte dem Lernenden vermittelt werden. Betrachtet man Schulungssysteme auf dieser Ebene, so muß also entschieden werden, wie das System aufgebaut sein soll. Relevante Fragen sind beispielsweise, ob die Drehmaschinenbedienung anhand eines Videos, mittels einer textuellen Beschreibung oder durch Probebedienung des Lernenden unter Aufsicht eines Experten geschult wird. Im Falle der Textverarbeitung könnte man etwa überlegen, ob man ein Hilfesystem oder ein geführtes Tutorial einsetzt.

- Ebene 4 (Meta ${ }^{2}$-Ebene): Blickt man ,von oben“ auf die in Ebene 3 betrachteten Schulungskonzepte, so gelangt man zu Fragestellungen der MetaSchulung. Auf dieser Ebene ist somit die Schulung des Schulungssystems Betrachtungsgegenstand. Hierunter sind dann z.B. eine Dokumentation der Bedienung des Schulungsvideos zu Drehmaschinen oder eine Anleitung „Wie benutze ich die Online-Hilfe zum Textverarbeitungssystem?“ zu subsumieren.

Die Relevanz der dargestellten Ebenen zeigt sich am Beispiel von KIWI. Bei der Erstellung des Systems standen die Schulungsinhalte im Mittelpunkt. Ausgehend von Ebene 2 wurde daher überlegt, in welcher Form die zu vermittelnden Inhalte (Prozeßorientierung, Workflow Management, KVP) schulungsmäßig gestaltet werden können. Damit stellte sich die Frage nach den zu berücksichtigenden Schulungskonzepten der Ebene 3. Die Entscheidung fiel hierbei für die in Abschnitt 2.3 beschriebenen und in Abbildung 7 aufgeführten Konzepte. So wurden die einzelnen Lerninhalte mit den Schulungskonzepten verknüpft und in eine geeignete Form gebracht. Ergebnis dieser Bemühungen sind z.B. eine interaktive Fallstudie zum Thema Workflow Management, eine Demonstration einer KVPZirkel-Sitzung, eine geführte Tour quer durch alle Themengebiete etc. Der Schwerpunkt der Entwicklung lag damit in den Ebenen 2 und 3. Zusätzlich macht eine Hilfefunktion dem Lernenden klar, wie mit KIWI umzugehen ist (Ebene 4).

Bei der Einführung von KIWI beim Anwenderpartner fischer [2] im Verlauf des MOVE-Projekts wurde klar, daß ein erhöhter Anwendungsnutzen erst dann entsteht, wenn die allgemeinen Lerninhalte ergänzt werden um anwendungs- und ausprägungsspezifische Aspekte. So entsteht für den Workflow-Anwender dann der größte Nutzen, wenn er im Sinne einer Benutzungsdokumentation Informationen zu Workflow-Typen und -Instanzen abrufen kann. Daher wurde für den Workflow Vorkalkulation bei fischer eine Dokumentation auf Typebene erstellt, über welche die Mitarbeiter den Umgang mit den erforderlichen Masken und 
Dokumenten erlernen können. Abbildung 8 zeigt die Dokumentation von Maskeneingaben für den Workflow-Typ Vorkalkulation.

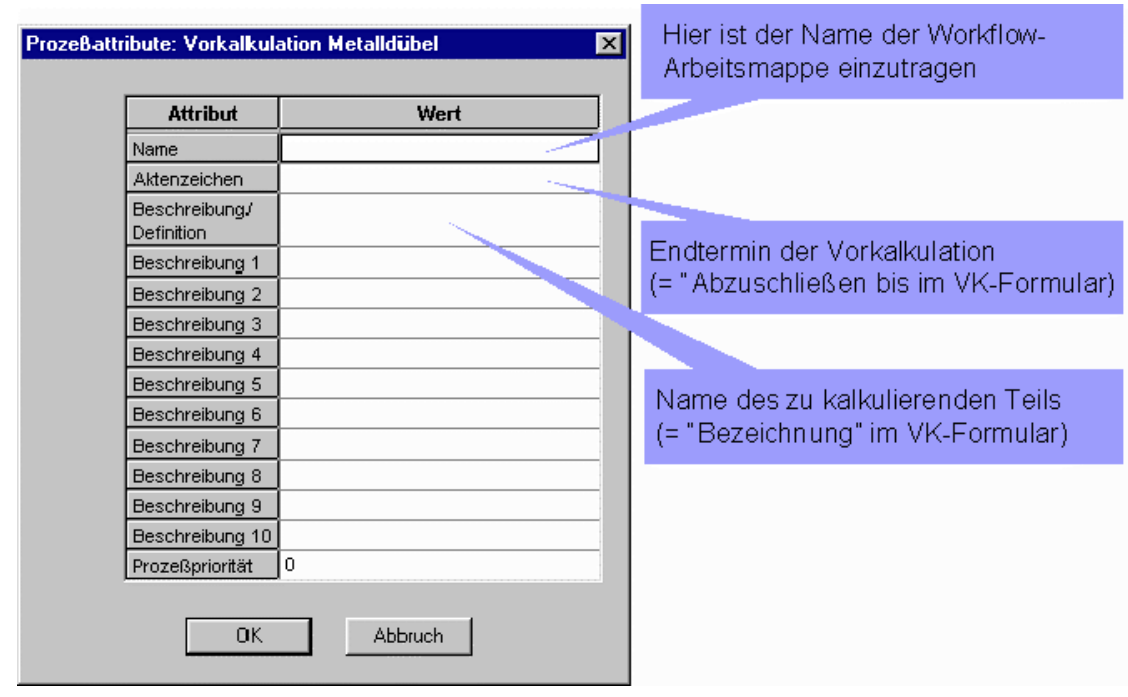

Abbildung 8: Benutzungsdokumentation auf Typebene

Für die zu pflegenden Eingabefelder ist jeweils dargestellt, in welcher Form die Eintragungen erfolgen sollten. Die Benutzungsdokumentation auf der Ebene von Ausprägungen war bisher noch kein Thema, könnte aber im weiteren Projektverlauf durchaus interessant werden, um insbesondere Sonderfälle der Bearbeitung zu schulen (Goesmann et al. 1998). Bezogen auf Abbildung 8 könnte eine Dokumentation auf Ausprägungsebene z.B. beinhalten, daß im Feld Prozeßpriorität (unterstes Eingabefeld) bei einer Vorkalkulation für den Kunden Meyer immer die höchste Priorität eingetragen werden muß, da es sich um einen äußerst wichtigen Kunden handelt.

\subsection{Betrachtungsobjekte workflow-bezogener Verbesserungsaktivitäten}

Als Grundlage zur Identifizierung von Objekten workflow-bezogener Verbesserungsaktivitäten eignet sich das von Scheer beschriebene allgemeine Geschäftsprozeßmodell (Scheer 1998, S. 31). In Abbildung 9 ist dieses um die interessierenden Dimensionen der Verbesserungsaktivitäten erweitert. 


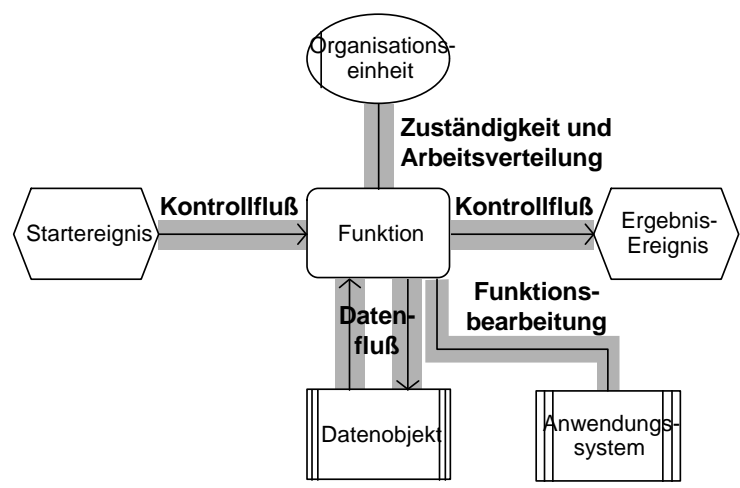

Abbildung 9: Dimensionen von Verbesserungsaktivitäten im allgemeinen Geschäftsprozeßmodell (in Anlehnung an Scheer 1998, S. 31)

Prozesse stehen beim Workflow Management im Mittelpunkt organisatorischer Gestaltungsentscheidungen wie sie beispielsweise im Rahmen von Verbesserungsaktivitäten getroffen werden. Um Prozesse kontinuierlich zu verbessern ist es allerdings keineswegs ausreichend, alleine den Ablauf, d.h. den K-ontrollfluß, zu analysieren. Vielmehr sind neben der reinen Prozeßlogik auch Aspekte wie der Datenfluß, organisatorische Zuständigkeiten und Arbeitsverteilung sowie die Funktionsbearbeitung von besonderem Untersuchungsinteresse. So beziehen sich Verbesserungsmaßnahmen zu Workflows in der Regel darauf, wie

- der reine Ablauf optimiert werden kann, z.B. durch Parallelisierung von Funktionen oder Eliminierung von Schleifen (Kontrollfluß),

- die zur Verfügung stehenden Daten optimal eingesetzt werden können, z.B. durch Datenübernahme aus operativen Systemen oder gemeinsamen Zugriff auf aktuelle Datenbestände (Datenfluß),

- Anwendungssysteme die durchzuführenden Funktionen optimal unterstützen können, z.B. durch Bereitstellen geeigneter Bearbeitungsmasken (Funktionsbearbeitung),

- die organisatorische Zuständigkeit entsprechend der Unternehmungsstruktur bestmöglich festgelegt werden kann, z.B. durch Bildung von Bearbeiterpools (Zuständigkeit),

- zur Laufzeit die konkrete Verteilung von Arbeitsobjekten an den einzelnen Mitarbeiter zielgerichtet vorgenommen werden kann, z.B. durch Abgleich von Qualifikations- und Anforderungsprofilen (Arbeitsverteilung) (Hagemeyer et al. 1998a).

Neben den aus quantitativem Datenmaterial (Durchlaufzeiten, Prozeßkosten, Mitarbeiterauslastungen u.a.) ableitbaren Verbesserungsmöglichkeiten nehmen auf dem Wissen und der Erfahrung der Anwender beruhende Verbesserungsvorschläge eine zunehmend wichtige Rolle ein. Um den Anwender bei der Ideenfindung, -formulierung und -umsetzung möglichst gut zu unterstützen, sind Werkzeuge wie etwa KIWI von großer Bedeutung. Dies wurde in den vorangegangenen Abschnitten bereits klar formuliert. Wichtig ist allerdings, dabei zu beachten, daß die 
oben aufgeführten Dimensionen von Verbesserungsaktivitäten unterschiedliche Auswirkungen auf die Gestaltung solcher Werkzeuge haben. So ist davon auszugehen, daß ein Mitarbeiter seine Verbesserungsideen nicht immer auf dieselbe Weise dokumentieren möchte. Je nach Art der Idee und des Lösungsvorschlags kommen unterschiedliche Medien in Betracht. Auf der Ebene des Kontrollflusses ist dem Anwender ohne weiteres zuzutrauen, daß er einen Verbesserungsvorschlag in Form eines überarbeiteten Modells abgibt. Eine entsprechende Schulung der verwendeten Modellierungsmethode wie EPK oder Petri-Netz ist natürlich sicherzustellen. Beziehen sich die Ideen des Anwenders allerdings auf Aspekte, die über die reine Prozeßlogik hinausgehen, so bieten die existierenden Workflow-Modelle sicherlich keine geeignete Visualisierung. Screen-Shots von Bearbeitungsmasken beispielsweise sind eine wesentlich bessere Grundlage, um Vorschläge zur Funktionsbearbeitung oder auch zum Datenfluß zu spezifizieren. Möchte man für eine laufende Prozeßinstanz die Arbeitsverteilung optimieren, so liefern WorkflowModelle keinerlei relevante Informationen. Erst statistische Betrachtungen liefern Ansatzpunkte für erforderliche Umverteilungsmaßnahmen.

Es läßt sich somit konstatieren, daß im Rahmen eines workflow-bezogenen Verbesserungsmanagements vielfältige Techniken erforderlich sind, um die aus der Mitarbeiterkreativität resultierenden Ideen möglichst gut abzubilden. Werkzeuge zur Unterstützung derartiger Aktivitäten müssen geeignete Konstrukte bereitstellen, um nicht wiederum selbst als Hemmnis aufgefaßt zu werden. Viele der genannten Anforderungen wurden in KIWI bereits umgesetzt. Weitere Ansätze werden derzeit auf mögliche Realisierungsalternativen im Betrieb überprüft.

\section{Fazit}

Im vorliegenden Beitrag wurde dargestellt, auf welche Art und Weise sich Workflow Management, organisatorisches Lernen und kontinuierlicher Verbesserungsprozeß in der betrieblichen Praxis gegenseitig ergänzen können. Voraussetzung hierfür ist die Schaffung einer geeigneten Infrastruktur wie sie durch ein Intranet gewährleistet ist. Durch Zugriff auf aktuelle und vor allem schnell aktualisierbare Schulungsinhalte können Mitarbeiter einen stetigen Lernprozeß arbeitsbegleitend durchlaufen. Dieser qualifiziert sie für die Nutzung neuartiger Technologien wie Workflow-Management-Systemen. Da beim Workflow Management allerdings die Geschäftsprozesse nicht im einmal erreichten Zustand erstarren sollten, sind Methoden des KVP von Bedeutung, die beim Anwender sowohl das Grundverständnis für ständige Weiterentwicklungsprozesse wecken als auch ihn zur Durchführung von Verbesserungsprozessen befähigen. Insofern sind organisatorisches Lernen und KVP sehr eng miteinander verwandt, da nur solche Unternehmungen sich organisatorisch weiterentwickeln, deren Mitarbeiter neu erlangtes individuelles Wissen ständig in Form von Verbesserungsideen wieder an die Unternehmung zurückgeben und so zum Anwachsen des organisationalen Wissensbestands beitragen. 
Informationssysteme zur Unterstützung eines derartigen Wissenstransformationsprozesses müssen sorgfältig gestaltet werden. Eine enge Verknüpfung von Schulung und Ideenmanagement scheint hierbei vielversprechend. Diese war bei der Erstellung des Prototyps KIWI oberste Maxime. Nur wenn der Mitarbeiter gerade erlernte Konzepte, Verhaltens- und Vorgehensweisen unmittelbar in seinem Arbeitsleben (erfolgreich) anwenden kann, wird der nötige Lernaufwand gerne in Kauf genommen. Aus diesem Grund ist es erforderlich, sich im Vorfeld der Entwicklung von Schulungs- und Verbesserungssystemen Gedanken über die spätere Ausrichtung des Systems zu machen. Zur Einordnung von Schulungssystemen und der Relevanz unterschiedlicher Betrachtungsobjekte bezüglich der Prozeßverbesserung wurden hierzu in diesem Beitrag einige Gedanken vertieft.

\section{Anmerkungen}

[1] Fördernummer 01 HB 9606/1. Informationen zu diesem Projekt können im WWW unter http://www.do.isst.fhg.de/move oder http://www.iwi.unisb.de/move abgerufen werden.

[2] Die fischer Holding GmbH \& Co. KG (Tumlingen/Waldachtal) ist Anwenderpartner im Forschungsprojekt MOVE. Erste Erfahrungen mit der Workflow-Einführung und -Nutzung sowie Schulung und KVP wurden hier im Geschäftsprozeß Vorkalkulation gemacht.

\section{Literaturverzeichnis}

Döge, M. (1997): Intranet: Einsatzmöglichkeiten, Planung, Fallstudien. 1. Aufl., Köln 1997.

Eipper, M. (1998): Sehen - Erkennen - Wissen: Arbeitstechniken rund um Mind Mapping. Renningen-Malmsheim 1998.

Gaitanides, M./Scholz, R./Vrohlings, A. (1994): Prozeßmanagement - Grundlagen und Zielsetzungen. In: Gaitanides, M. et al. (Hrsg.): Prozeßmanagement: Konzepte, Umsetzungen und Erfahrungen des Reengineering. München et al. 1994, S. 2-19.

Goesmann, T./Just-Hahn, K./Löffeler, T./Rolles, R. (1998): Unterstützung von Sonderprozessen durch Workflow-Management-Systeme. In: Proceedings zum Workshop „Flexibilität und Kooperation in WorkflowManagement-Systemen“, D-CSCW '98. In Veröffentlichung.

Hagemeyer, J./Rolles, R. (1998): Erhebung von Prozeßwissen für das Wissensmanagement. IM Information Management \& Consulting 13 (1998) 1, S. 4650.

Hagemeyer, J./Rolles, R./Schmidt, Y./Scheer, A.-W. (1998a): Arbeitsverteilungsverfahren in Workflow-Management-Systemen: Anforderungen, Stand und Perspektiven. In: Scheer, A.-W. (Hrsg.): Veröffentlichungen des Instituts für Wirtschaftsinformatik, IWi-Heft 145, Saarbrücken 1998. 
Hagemeyer, J./Rolles, R./Schmidt, Y./Bachmann, J./Haas, A. (1998b): Dynamische Prozesse durch workflow-zentrierte Geschäftsprozeßoptimierung: Herausforderungen in der Praxis. In: Scheer, A.-W. (Hrsg.): Neue Märkte, neue Medien, neue Methoden - Roadmap zur agilen Organisation. 19. Saarbrücker Arbeitstagung, Heidelberg 1998.

Herrmann, Th./Scheer, A.-W./Weber, H. (Hrsg.) (1998a): Verbesserung von Geschäftsprozessen mit flexiblen Workflow-Management-Systemen: Von der Erhebung zum Sollkonzept. Heidelberg 1998.

Herrmann, Th./Scheer, A.-W./Weber, H. (Hrsg.) (1998b): Verbesserung von Geschäftsprozessen mit flexiblen Workflow-Management-Systemen: Von der Sollkonzeptentwicklung zur Implementierung von WorkflowManagement-Anwendungen. Heidelberg 1998.

Imai, M. (1994): Kaizen - Der Schlüssel zum Erfolg der Japaner. 12. Aufl., München 1994.

Ishikawa, K. (1985): What is quality control? The Japanese way. Englewood Cliffs, N.J. 1985.

Jacobi, J.-M. (1997): Kontinuierlich verbessern: Jeder kann kreativ sein: Das neue BVW. 2. Aufl., Stuttgart 1997.

Jain, R. (1997): Visual Information Management. Communications of the ACM 40 (1997) 12, S. 31-32.

Mehrmann, E. (1994): Schnell am Ziel: Kreativitäts- und Problemlösungstechniken. Düsseldorf et al. 1994.

Oberschulte, H.(1994): Organisatorische Intelligenz - ein integrativer Ansatz des organisatorischen Lernens. München et al. 1994.

Rolles, R. (1998): Kontinuierliche Verbesserung von workflow-gestützten Geschäftsprozessen. In: Herrmann, Th./Scheer, A.-W./Weber, H. (Hrsg.): Verbesserung von Geschäftsprozessen mit flexiblen Workflow-Management-Systemen: Von der Erhebung zum Sollkonzept. Heidelberg 1998, S. 109-133.

Scheer, A.W. (1998): ARIS - Vom Geschäftsprozeß zum Anwendungssystem. 3. Aufl., Berlin et al. 1998.

Schnauber, H./Grabowski, S./Schlaeger, S./Zülch, J. (1997): Total Quality Learning: Ein Leitfaden für lernende Unternehmen. Berlin et al. 1997.

Thom, N. (1991): Betriebliches Vorschlagswesen - Ein Instrument der Betriebsführung: Empirische Erkenntnisse und Gestaltungsempfehlungen. 4. Aufl., Bern et al. 1991.

Von Krogh, G./ Venzin, M. (1995): Anhaltende Wettbewerbsvorteile durch Wissensmanagement. Die Unternehmung 49 (1995) 6, S. 417-436.

Walter, T. (1998): Visualisierungsmethoden bei Workflow-Management - Prototyping und Showcases. In: Proceedings der DCSCW '98, in Veröffentlichung. 